\title{
Hybrid selection for sequencing pathogen genomes from clinical samples
}

\author{
Alexandre Melnikov ${ }^{1}$, Kevin Galinsky ${ }^{1}$, Peter Rogov ${ }^{1}$, Timothy Fennell ${ }^{1}$, Daria Van Tyne ${ }^{2}$, Carsten Russ ${ }^{1}$, \\ Rachel Daniels' ${ }^{1}$ Kayla G Barnes², James Bochicchio ${ }^{1}$, Daouda Ndiaye ${ }^{3}$, Papa D Sene ${ }^{3}$, Dyann F Wirth², \\ Chad Nusbaum', Sarah K Volkman², Bruce W Birren ${ }^{1}$, Andreas Gnirke ${ }^{1}$ and Daniel E Neafsey ${ }^{1 *}$
}

\begin{abstract}
We have adapted a solution hybrid selection protocol to enrich pathogen DNA in clinical samples dominated by human genetic material. Using mock mixtures of human and Plasmodium falciparum malaria parasite DNA as well as clinical samples from infected patients, we demonstrate an average of approximately 40 -fold enrichment of parasite DNA after hybrid selection. This approach will enable efficient genome sequencing of pathogens from clinical samples, as well as sequencing of endosymbiotic organisms such as Wolbachia that live inside diverse metazoan phyla.
\end{abstract}

\section{Background}

The falling cost of DNA sequencing means that sample quality, rather than expense, is now the blocking issue for many infectious disease genome sequencing projects. Pathogen genomes are generally very small relative to that of their human host, and are typically haploid in nature. Therefore, even a modest number of nucleated human cells present in infectious disease samples may result in the pathogen DNA representation being dwarfed relative to the host human DNA. This difference in representation poses a significant challenge to achieving adequate sequence coverage of the pathogen genome in a cost-effective manner. Separation of host and pathogen cells prior to DNA extraction can be difficult or inconvenient, particularly in field settings common to clinics in developing countries.

This barrier to the efficient sequencing of pathogen genomes comes at a time when the potential motivations and rewards for large-scale sequencing of pathogens are becoming increasingly clear. Examples abound to demonstrate how whole-genome analyses of pathogen population structure from large numbers of isolates can help to identify the source of disease outbreaks or hidden subpopulations. Whole genome sequencing of 35 Salmonella enterica samples was recently performed by

\footnotetext{
* Correspondence: neafsey@broadinstitute.org

'Genome Sequencing and Analysis Program, Broad Institute, 7 Cambridge Center, Cambridge, MA 02142, USA Full list of author information is available at the end of the article
}

the United States Food and Drug Administration in order to identify the source of a foodborne illness outbreak that affected approximately 300 individuals in 2009 and 2010 [1]. Whole genome sequencing of 20 isolates of pathogenic Coccidiodies spp. fungi identified gene flow in select genomic regions between the recently diverged Coccidiodies immitis and Coccidiodies posadasii [2]. Whole genome sequencing and comparative SNP analysis of unculturable Mycobacterium leprae isolates was utilized to demonstrate that a third of leprosy infections in the United States derive from armadillos [3]. So-called 'third generation' sequencing was successfully employed to identify the origin of the recent Haitian cholera outbreak strain via de novo sequencing of 5 isolates and comparison of those sequences to 23 previously sequenced isolates of Vibrio cholera [4]. In addition, the increasing use of genomewide association studies to determine the genetic basis of important infectious disease phenotypes, such as drug resistance in malaria parasites $[5,6]$, will require sequencing or genotyping hundreds to thousands of pathogen isolates, making a shortage of quality specimens an acute problem. All of these studies could have been performed more expediently if a culturing step were not required to eliminate DNA derived from the host or environment.

Existing methods for dealing with DNA contamination in infectious disease samples typically require significant time, money, and/or special handling of samples at the
C Biomed Central 
time of collection. Taking Plasmodium falciparum as a model case, malaria parasite samples in blood may be adapted to in vitro culture and sustained in a pure medium of DNA-free human red blood cells. The adaptation process, however, can take more than 6 weeks, requires considerable expertise and expense [7], and may potentially select for culturable variants. To remedy this, DNA-containing white blood cells may be depleted directly from malaria patient blood samples prior to cell lysis via differential density centrifugation or column filtration [8-10]. While depletion methodologies may reduce white cell abundance to levels useful for biochemical assays, the 100-fold disparity in genome size between human and malaria means that an even modest number of host cells can compromise a sample for genome sequencing. In addition, white blood cell depletion currently requires a significant volume of blood to be drawn from patients (approximately $5 \mathrm{ml}$ ), and the blood must then be stored at minus $70^{\circ} \mathrm{C}$ in a special medium to preserve cellular integrity. This could preclude sample collection for genome sequencing from many clinical trials due to protocol limitations or lack of equipment in the field. Furthermore, pathogens that infect or closely associate with nucleated host cells, such as Plasmodium vivax, Trypanosoma cruzi, or Chlamydia trachomatis, are not amenable to purification by white cell depletion. Endosymbionts such as Wolbachia, which influence host fertility and other traits in filarial worms, insect disease vectors, and diverse other taxa, may only be cultured in an intracellular system [11], precluding easy isolation of their genomic DNA for sequencing except by elaborate methods [12].

To address this problem we have adapted a solution hybrid selection approach originally developed for the purification of resequencing targets in the human genome [13]. In brief, biotinylated RNA probes complementary to the pathogen genome ('baits') are hybridized to pathogen DNA in solution and pulled down with magnetic streptavidin-coated beads. Host DNA is washed away and the captured pathogen DNA may then be eluted and amplified for sequencing or genotyping. We experimented with two approaches to bait design: synthetic 140 -bp oligos targeting specific regions of the $P$. falciparum 3D7 reference genome assembly and 'whole genome baits' (WGBs) generated from pure P. falciparum DNA. Using this protocol, we achieved significant enrichment of $P$. falciparum DNA, to a level that allowed us to conduct whole genome sequencing on samples that otherwise would have been prohibitively expensive to sequence.

\section{Results and discussion}

Hybrid selection on a mock clinical malaria sample

We performed hybrid selection with both classes of bait on a mock clinical sample consisting of $99 \%$ human
DNA and $1 \%$ Plasmodium DNA by mass, which falls within the range of DNA ratios found in many malaria clinical samples (Table 1). Hybridization and washing steps (see Materials and methods) were carried out under standard high stringency conditions to reduce capture of host DNA. The hybrid selection protocol requires a minimum of $2 \mu \mathrm{g}$ of input DNA (combined host and pathogen), a quantity that may not be available from many types of field samples. Therefore, we also performed hybrid selection with both bait classes on 2 $\mu \mathrm{g}$ of whole genome amplified DNA generated from 10 ng of the mock clinical sample. Quantitative PCR (qPCR) analysis indicated that whole genome amplification (WGA) does not significantly alter the fraction of malaria DNA present in the sample (post-WGA percentage $P$. falciparum DNA $=1.1 \pm 0.1$ ).

Sequencing of the hybrid-selected samples revealed a significant increase in representation of Plasmodium DNA in every case. The synthetic baits respectively yielded an average of 41-fold and 44-fold parasite DNA enrichment for unamplified and WGA simulated clinical samples in genomic regions targeted by the baits, as measured by qPCR. Whole genome baits yielded parasite genome-wide average enrichment levels of 37-fold and 40-fold for the unamplified and WGA input samples, respectively.

Illumina sequencing coverage in the WGB hybridselected samples is correlated with GC content, mirroring what is observed in sequencing data from pure $P$. falciparum DNA (Figure 1a). With a genome-wide A/T composition of $81 \%$ [14], achieving uniform sequencing coverage of the $P$. falciparum genome is challenging even under ideal circumstances. Despite this challenge, we observed no reduction in coverage uniformity as a result of the hybrid selection process. WGA did not compromise mean genome-wide sequencing coverage relative to unamplified input DNA $(67.5 \times$ versus $67.1 \times$ for a single Illumina GAIIx lane, respectively). Sequencing coverage of the samples hybrid selected using synthetic 140-bp baits was tightly localized to the genomic regions to which baits were designed (Figure 1b). Coverage levels in baited regions were significantly higher than the levels observed from comparable sequencing of pure $P$. falciparum DNA (mean coverage $=143.8 \times$ and $92 \times$, respectively; Wilcoxon rank sum test, $\mathrm{W}=6.7 \mathrm{E} 12$, $P<2.2 \mathrm{e}-16)$. This indicates that hybrid selection with synthetic baits may be useful not only for reducing offtarget coverage in the host genome, but also for strategically augmenting coverage levels in regions of pathogen genomes where heightened sequence coverage could be informative, such as highly polymorphic antigenic regions subject to host immune pressure.

Though effective sequencing coverage levels are reduced in the hybrid-selected mock clinical samples 
Table 1 Quantitative PCR enrichment measurements from 12 clinical samples

\begin{tabular}{|c|c|c|c|c|c|}
\hline \multirow[b]{2}{*}{ Sample } & \multirow{2}{*}{$\begin{array}{c}\text { Percentage } \\
\text { parasite DNA }\end{array}$} & \multirow[b]{2}{*}{ WGA } & \multicolumn{2}{|c|}{ 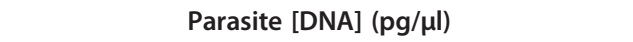 } & \multirow[b]{2}{*}{ Fold enrichment } \\
\hline & & & Pre-hybrid selection $^{a}$ & Post-hybrid selection $^{a}$ & \\
\hline Th231.08 (round 1) & 0.11 & Yes & $1.8(0.6)$ & $71.1(5.6)$ & 39.7 \\
\hline Th231.08 (round 2) & 7.7 & No & $71.1(5.6)$ & $349.1(74.9)$ & 4.9 \\
\hline Th145.08 & 20 & No & $198.4(17.4)$ & $477.6(66.7)$ & 2.4 \\
\hline Th032.09 & 12 & No & $114.7(2.9)$ & $372.6(59.3)$ & 3.2 \\
\hline Th029.09 & 3 & No & $33.6(0.8)$ & $317.3(54.7)$ & 9.4 \\
\hline Th093.09 & 2.8 & No & $28.5(1.5)$ & $365.6(53.4)$ & 12.8 \\
\hline Th090.08 & 2.3 & No & $37.7(1.1)$ & $300.4(46.9)$ & 8.0 \\
\hline Th139.08 & 2.1 & No & $23.6(0.6)$ & $346.2(50.7)$ & 14.7 \\
\hline Th197.08 & 1.1 & No & $14.6(0.0)$ & $222.7(36.1)$ & 15.3 \\
\hline Th140.08 & 0.99 & No & $9.6(0.1)$ & $251.5(37.4)$ & 26.2 \\
\hline Th190.08 & 0.64 & No & $5.1(0.2)$ & $218.7(34.0)$ & 43.2 \\
\hline Th238.08 & 0.53 & No & $6.7(0.2)$ & $273.4(38.1)$ & 41.0 \\
\hline Th127.09 & 1.6 & No & $26.8(0.4)$ & $368.5(57.1)$ & 13.7 \\
\hline Th175.08 & 48 & Yes & $275.8(7.2)$ & $556.9(79.4)$ & 2.0 \\
\hline
\end{tabular}

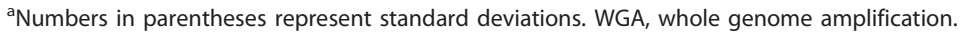

relative to pure $P$. falciparum DNA due to the incomplete elimination of human DNA, this reduction is small compared to the 100 -fold reduction in coverage expected without hybrid selection. Genome-wide coverage is depicted in Figure 2a, which illustrates that the extent of the genome covered to various thresholds is highly similar for the pure $P$. falciparum and hybridselected mock clinical samples, and significantly higher than simulated coverage levels we would have predicted to have observed from sequencing an un-purified version of the sample. Genome-wide coverage levels as a function of the local \%GC (the percentage of nucleotides in the genome that are $\mathrm{G}$ or $\mathrm{C} ; \% \mathrm{G}+\mathrm{C}$ ) are plotted in Figure $2 \mathrm{~b}$ for the WGB experiments. The relationship between \%GC and coverage observed in whole genome shotgun sequencing data is decreased by hybrid selection due to reduced coverage in rare high \%GC genomic regions (Spearman's $r_{s}$ for \%GC versus coverage of pure malaria DNA, 0.86; versus WGB hybrid-selected DNA, 0.59; versus WGA + WGB hybrid-selected DNA, 0.64). The vertical line in Figure $2 \mathrm{~b}$ represents the average \% GC of exonic sequence (23\%). Assuming a minimum threshold of 10-fold sequencing coverage is required for accurate SNP calling, 99.2\% of exonic bases exhibited this coverage or greater in reads generated from the pure $P$. falciparum DNA sample. The unamplified and amplified hybrid-selected samples achieved at least 10fold coverage for $98.3 \%$ and $98.0 \%$ of exonic bases, respectively. Given that previous pathogen population genomic analyses of outbreaks or population structure have been SNP-based $[1,2,4]$, this indicates that sequencing data generated from hybrid-selected clinical samples could be as useful as data generated from pure pathogen DNA samples for downstream analyses.
Further comparison of sequencing coverage between hybrid-selected and pure P. falciparum DNA indicates that local \%GC and polymorphism rate do not significantly influence sequencing coverage in a hybridselected sample (Additional file 1).

We attempted to optimize our hybrid selection protocol by exploring two different hybridization temperatures $\left(60^{\circ} \mathrm{C}\right.$ versus $\left.65^{\circ} \mathrm{C}\right)$ and four different 10 -minute wash stringencies $(0.1 \times \mathrm{SSC}, 0.25 \times \mathrm{SSC}, 0.5 \times \mathrm{SSC}$, and $0.75 \times$ SSC). Eight mock clinical samples were hybridized with WGB and washed under all combinations of the above conditions. Enrichment was measured by qPCR and sequencing (one indexed Illumina GAIIx lane). We observed the best enrichment under the standard high stringency conditions used for all previously reported experiments (hybridization at $65^{\circ} \mathrm{C}$ and high stringency wash $(0.1 \times$ SSC). Results are presented in Table 2 .

In summary, both bait strategies performed effectively and now offer investigators a method to sequence either targeted regions or complete genomes of pathogens in clinical samples dominated by host DNA. Pairing this hybrid selection protocol with WGA further expands the range of clinical samples now eligible for efficient pathogen genome sequencing. For example, for Plasmodium it should now be possible to sequence the parasite genome directly from dried blood spots on filter paper, an easily collectable and storable sample format.

\section{Hybrid selection on authentic clinical samples}

To test this application, we performed WGA and hybrid selection on DNA extracted from a clinical $P$. falciparum sample (Th231.08) collected on filter paper in Thies, Senegal in 2008 and stored at room temperature for over a year. By qPCR we estimated the Plasmodium 


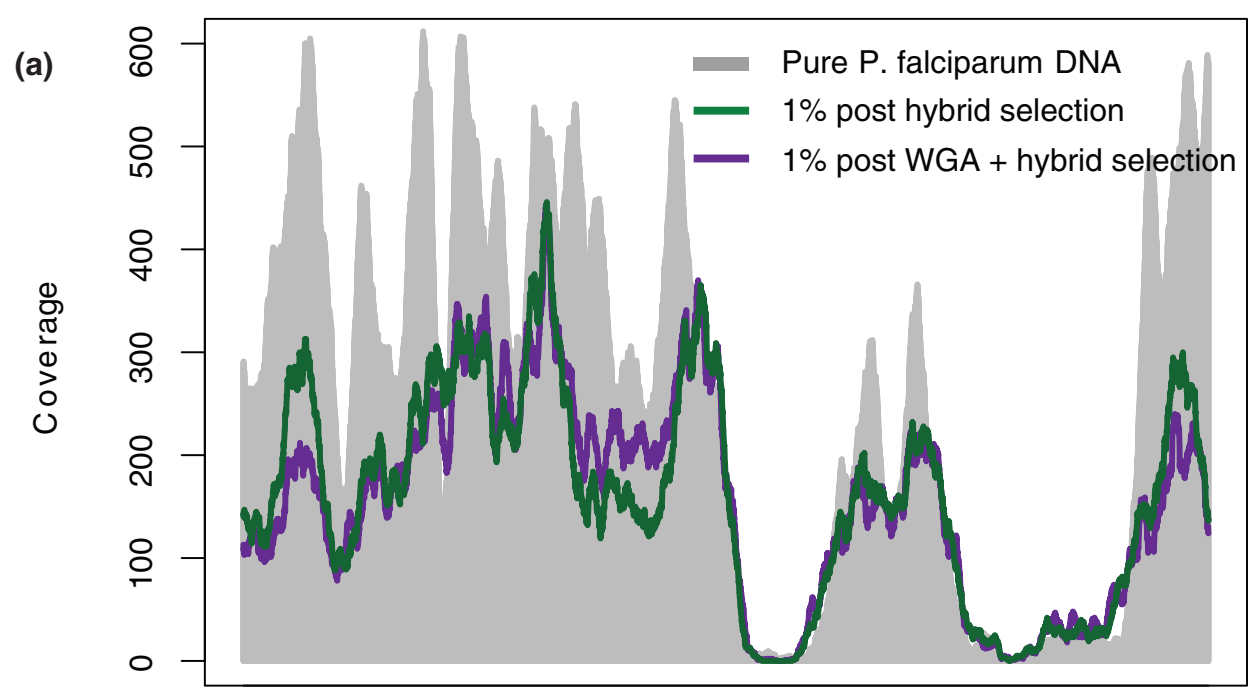

(b)

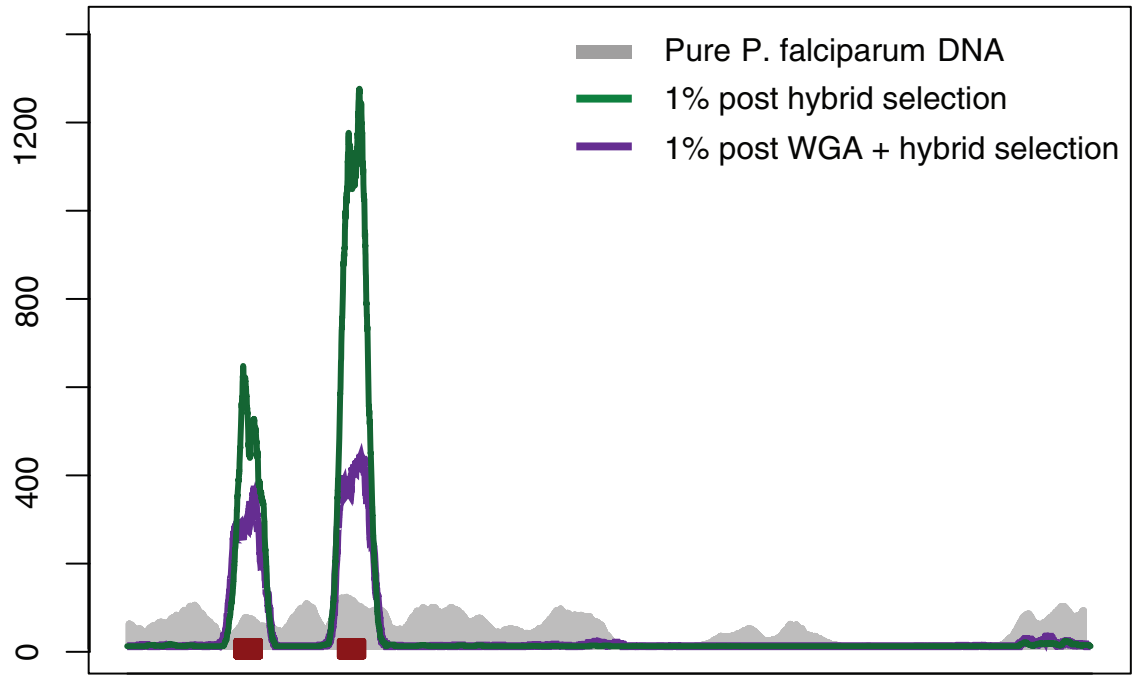

(c)

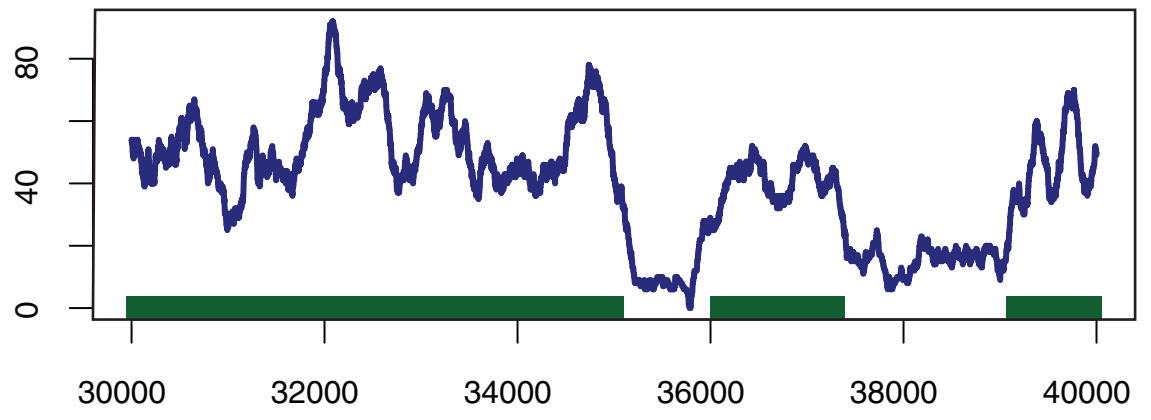

chr 1 position (bp)

Figure 1 Sequencing coverage plots from a randomly chosen region of $P$. falciparum chromosome 1. (a) Unamplified (green) and WGA (purple) WGBs compared to pure P. falciparum (gray). (b) Unamplified (green) and WGA (purple) synthetic bait read coverage compared to pure P. falciparum (gray). Red bars indicate bait locations. (c) Local \%GC (the percentage of nucleotides in the genome that are G or C; in 140-bp windows). Green bars indicate exons. Chr, chromosome. 
(a)

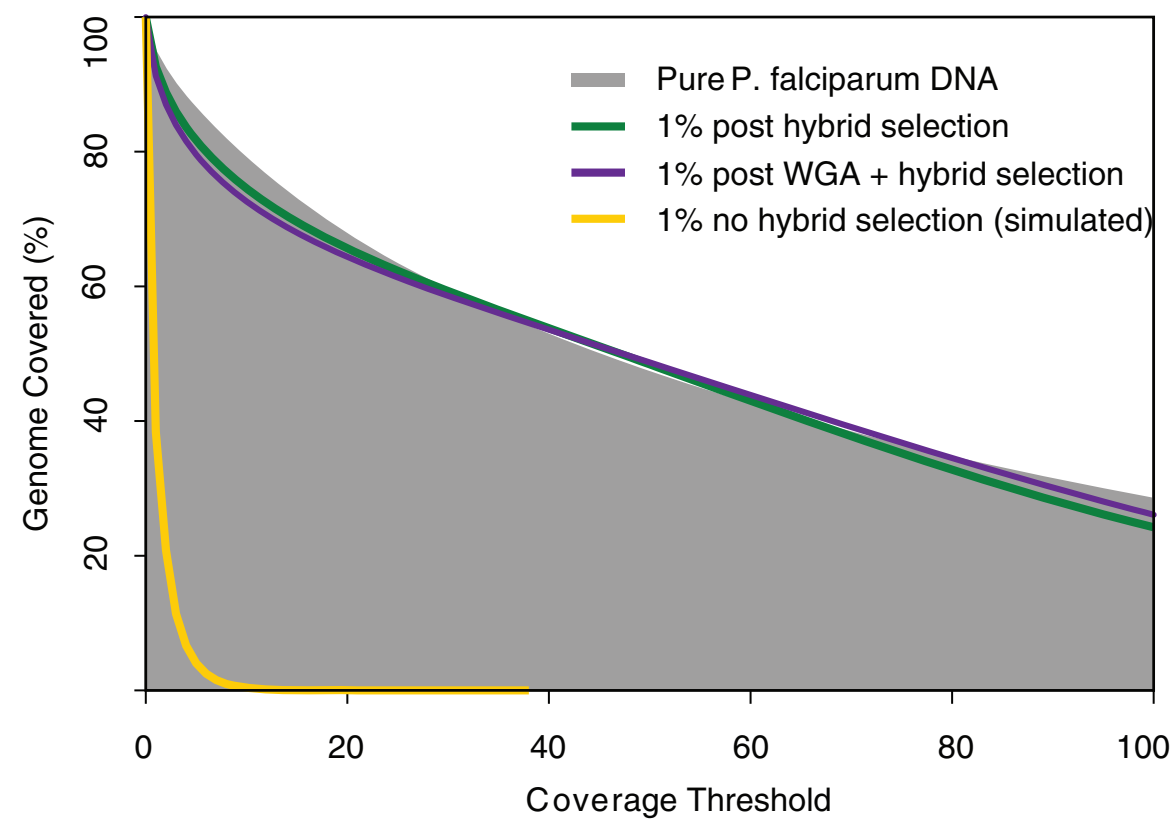

(b)

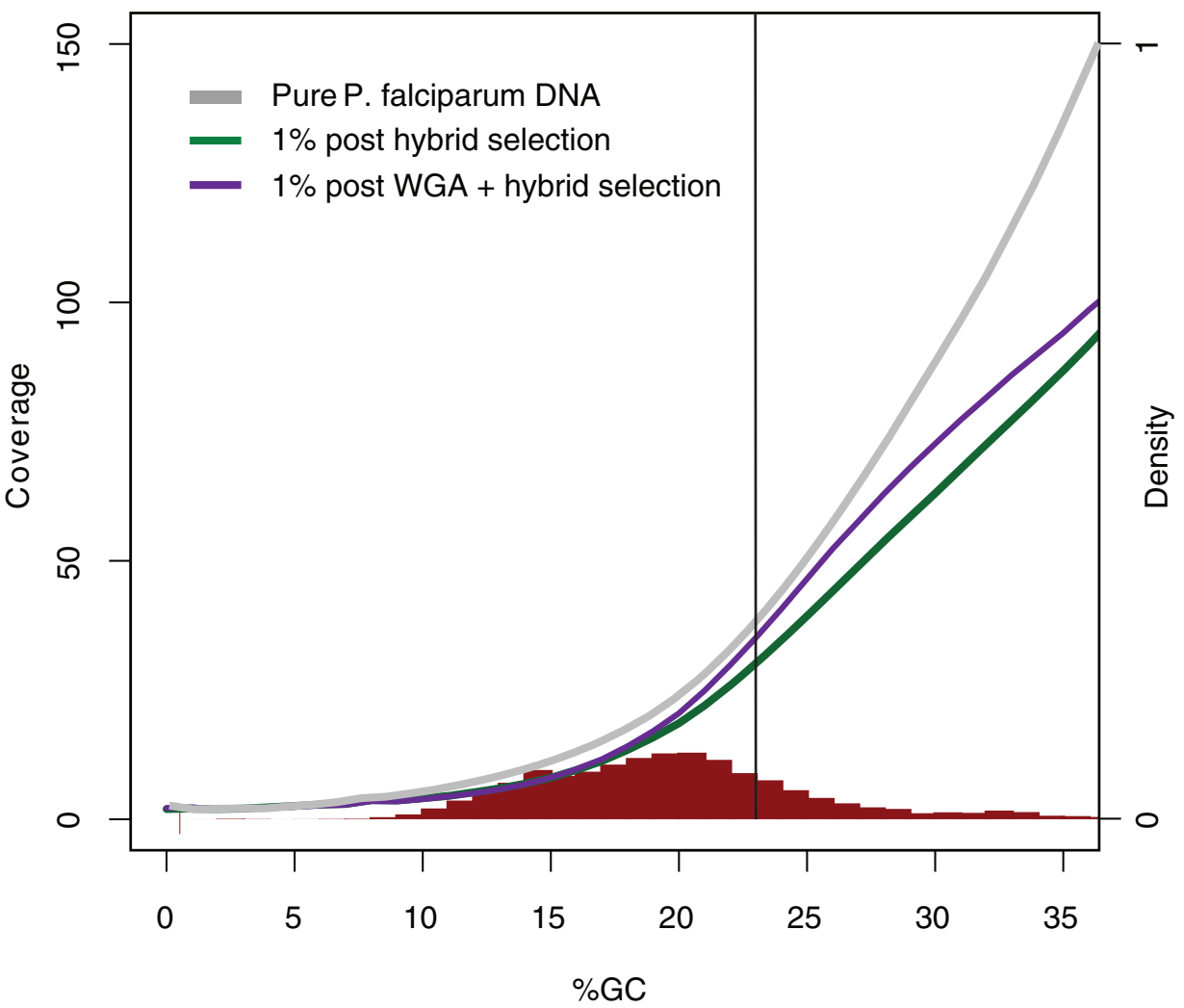

Figure 2 Genome-wide sequencing coverage and composition. (a) Coverage thresholds for unamplified (green) and WGA (purple) whole genome baits compared to pure P. falciparum (gray) and simulated coverage from a non-hybrid-selected mock clinical sample (yellow). (b) Genome-wide coverage as a function of \%GC. The vertical black line represents average exonic \%GC. The red histogram represents the probability density distribution of genome composition (right vertical axis). Lines depict coverage (left vertical axis) of pure $P$. falciparum DNA (gray), as well as unamplified (green) and WGA (purple) hybrid-selected samples initially containing $1 \%$ P. falciparum DNA. \%GC, the percentage of nucleotides in the genome which are $\mathrm{G}$ or $\mathrm{C}$. 
Table 2 Quantitative PCR enrichment measurements

\begin{tabular}{lllccc}
\hline Hybrid temperature & Stringency & Wash & $\begin{array}{c}\text { Pre-hybrid selection } \\
\text { [DNA] }(\mathbf{p g} / \mathbf{\mu l})\end{array}$ & $\begin{array}{c}\text { Post-hybrid selection } \\
\text { [DNA] }(\mathbf{p g} / \boldsymbol{\mu l})\end{array}$ & Fold enrichment \\
\hline $65^{\circ} \mathrm{C}$ & High & $0.10 \times$ SSC & 10.0 & 342.9 & 34.3 \\
& Med/high & $0.25 \times$ SSC & 10.0 & 258.2 & 25.8 \\
& Med/low & $0.50 \times$ SSC & 10.0 & 227.9 & 22.8 \\
$60^{\circ} \mathrm{C}$ & Low & $0.75 \times$ SSC & 10.0 & 181.4 & 18.1 \\
& High & $0.10 \times$ SSC & 10.0 & 288.6 & 28.9 \\
& Med/high & $0.25 \times$ SSC & 10.0 & 232.9 & 23.3 \\
& Med/low & $0.50 \times$ SSC & 10.0 & 203.5 & 196.3 \\
\hline
\end{tabular}

DNA in the original sample to comprise approximately $0.11 \%$ of the total DNA by mass. Following WGA and hybrid selection, Plasmodium DNA represented $7.7 \%$ of total DNA present, an approximately 70 -fold increase in parasite DNA representation. Illumina HiSeq sequencing data confirmed that at least $5.9 \%$ of mappable reads in the hybrid-selected sample corresponded to Plasmodium. The fraction of human reads after hybrid selection remained high due to the extreme initial ratio of host: parasite DNA, but the enrichment factor in this case was sufficient to rescue the feasibility of sequencing this sample. We evaluated the accuracy and utility of the data by calling SNPs against the P. falciparum reference assembly. We identified a total of 26,366 SNPs relative to the $P$. falciparum reference assembly (more than one per kilobase), close to the number of SNPs identified (33,094 to 41,123) from 11 other culture-adapted Senegalese parasite lines sequenced without hybrid selection. Further SNPs could likely be discovered by further augmenting coverage. While the depth of coverage we obtained from this experiment would not be sufficient for de novo genome assembly, SNP calling against a reference assembly is the end-stage analysis for most Illumina data (for example, [1-4]) and therefore a good indication of a dataset's potential utility. Principal components analysis of SNP genotypes confirms the similar genomic profile of the hybrid-selected and non-hybrid-selected Senegalese strains, as well as hybrid-selected and non-hybridselected 3D7 reference strain datasets generated from sequencing the mock clinical samples (Figure 3). Despite the use of WGBs generated from the 3D7 reference genome, the DNA captured from the Senegal isolate has the SNP profile of Senegal DNA, rather than 3D7 DNA, suggesting that polymorphisms do not strongly bias enrichment. In addition, the highly polymorphic regions of the isolate did not suffer a relative drop in sequencing coverage after hybrid selection. Selection of a panel of 12 other clinical malaria samples from Senegal yielded an average of 35 -fold enrichment, as measured by qPCR (Table 1), with enrichment amount inversely proportional to the initial fraction of parasite DNA in the samples.
We conducted a second round of hybrid selection on the Th231.08 clinical sample to determine whether the Plasmodium DNA titer in the sample could be boosted above approximately $7 \%$. The second round of hybrid selection was carried out under identical hybridization and wash conditions. qPCR analysis indicates this yielded a sample in which $47.5 \%$ of the genetic material was Plasmodium by mass (a 6.7 -fold enrichment). This lower fold enrichment is consistent with our previous observation that fold enrichment is inversely proportional to initial parasite DNA titer, but in this case an additional round of hybrid selection yields a sample even more amenable to cost-efficient and deep sequencing.

Although sequencing has become considerably less expensive in recent years, it remains financially impractical

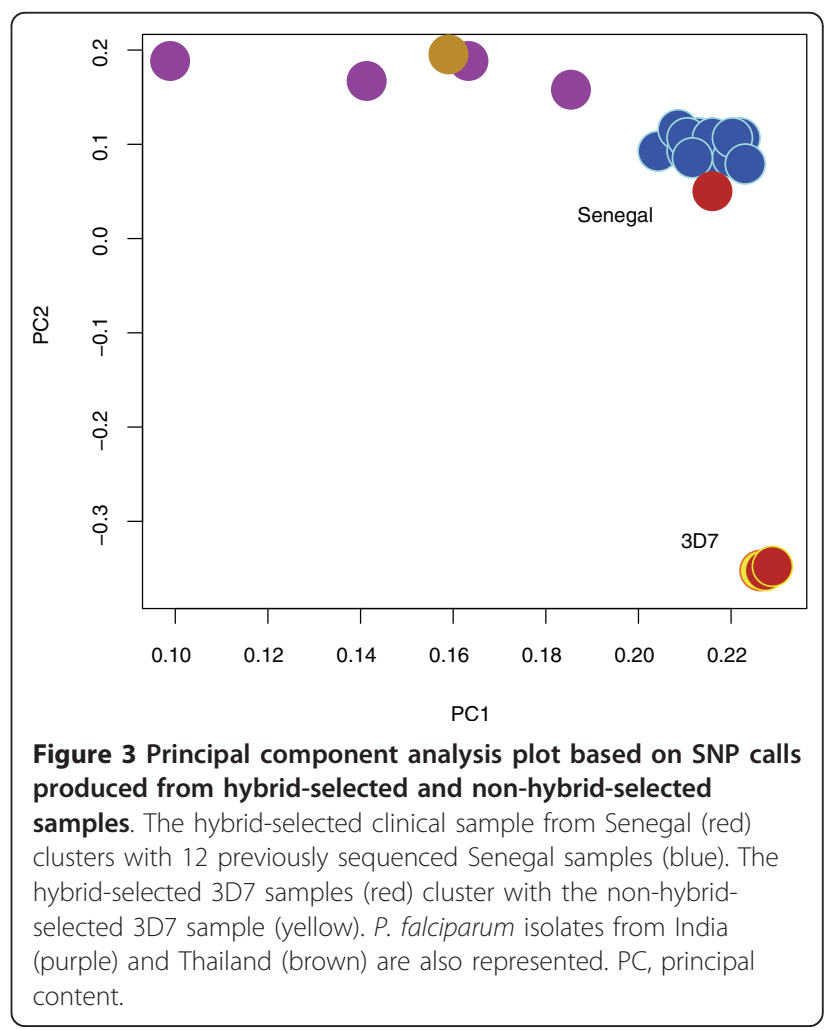


to sequence pathogen genomes from clinical samples at scale due to the gross excess of host DNA typically present. The simplest way to compensate for host DNA contamination is to augment sequencing coverage depth. However, this strategy can be costly for all but the most lightly contaminated samples. In contrast, the cost of purification by hybrid selection using whole genome baits is approximately US $\$ 250$, which is roughly equivalent to the current cost of generating 20-fold coverage of the $23 \mathrm{Mb} P$. falciparum genome from pure template using a fraction of an Illumina HiSeq lane. For augmented coverage to be an affordable strategy relative to hybrid selection for a target coverage level of $40 \times$ in a genome of this size, samples must contain at least $50 \%$ pathogen DNA. This titer of parasite DNA is rarely found in clinical samples unless white cell depletion is performed prior to DNA extraction. For a more typical clinical sample containing only $1 \%$ P. falciparum DNA, hybrid selection resulting in 40-fold enrichment enables 40 $\times$ coverage depth for a dramatically lower total price (approximately $\$ 1,000$ ) than deeper sequencing of the unpurified sample (approximately \$40,000).

\section{Conclusions}

The modest cost and high performance of this hybrid selection purification protocol will facilitate sequencing of archival clinical samples of malaria parasites and other pathogens previously considered unfit for sequencing by any methodology. This may enable sequencing of important samples stored on filter papers or diagnostic slides predating the spread of drug resistance or associated with historic outbreaks. This purification protocol also broadens the accessibility of sequencing for clinical samples of infectious organisms for which in vitro culture is possible but costly or inconvenient, such as class IV 'select agents' recognized by the Centre for Disease Control. This protocol is not limited to pathogens, and should be equally useful in sequencing commensal or symbiotic organisms closely associated with their host, such as intracellular Wolbachia bacteria, as was recently demonstrated by Kent et al. in their application of an array-based capture protocol [15]. The reduction in sample quality and quantity requirements permitted by hybrid selection will simplify protocol design in future large-scale clinical studies and help realize the benefits of inexpensive, massively parallel sequencing technologies for studying infectious diseases in diverse contexts.

\section{Materials and methods Samples}

Mock clinical samples were generated by mixing Homo sapiens NA15510 DNA with a pure preparation of $P$. falciparum 3D7 parasite DNA at a ratio of 99:1 $(H$. sapiens: $P$. falciparum) by mass. Samples were fluorescently quantified prior to mixing using a PicoGreen [16] assay. Authentic clinical samples were collected in 2008 from symptomatic patients at a clinic in Thies, Senegal under an approved institutional review board protocol. Samples consisted of whole blood dried and stored on a Whatman FTA card (fast technology for analysis of nucleic acids) and/or frozen whole blood stored in glycerolyte 57 solution. DNA was extracted using a DNeasy kit (Qiagen Hilden, North Rhine-Westphalia, Germany). Whole frozen blood samples yielded sufficient DNA for hybrid selection, but samples from FTA cards typically yielded less than $100 \mathrm{ng}$ of DNA and required WGA. WGA was performed using the Repli-G kit (Qiagen).

\section{Bait design and preparation}

Synthetic 140-bp oligos were obtained from Agilent and designed to capture exonic regions of the P. falciparum genome as defined in the 3D7 v.5.0 reference assembly. The final bait set included 24,246 oligos (3.4 Mb) with unique BLAT matches to the $P$. falciparum 3D7 reference genome assembly and no homology to the human genome. Baits and locations are listed in Additional file 2. To generate synthetic single-stranded biotinylated RNA bait, in vitro transcription was performed with biotin-labeled UTP using the MEGAshortscript T7 kit (Ambion Austin, Texas, United States) as described previously [13].

WGB was generated at the Broad Institute. For input, $3 \mu \mathrm{g}$ of $P$. falciparum 3D7 DNA was sheared for 4 minutes on a Covaris E210 instrument set to duty cycle 5, intensity 5 and 200 cycles per burst. The mode of the resulting fragment size distribution was $250 \mathrm{bp}$. End repair, addition of a 3'-A, adaptor ligation and reaction clean-up followed the Illumina's genomic DNA sample preparation kit protocol except that adapter consisted of oligonucleotides 5'-TGTAACATCACAGCATCACCGC CATCAGTCxT-3' ('x' refers to an exonuclease I-resistant phosphorothioate linkage) and 5'-[PHOS]GACTG ATGGCGCACTACGACACTACAATGT-3'. The ligation products were cleaned up (Qiagen), amplified by 8 to 12 cycles of PCR on an ABI GeneAmp 9700 thermocycler in Phusion High-Fidelity PCR master mix with HF buffer (NEB Ipswich, Massachusetts, United States) using PCR forward primer 5'-CGCTCAGCGGCCG CAGCATCACCGCCATCAGT-3' and reverse primer 5'CGCTCAGCGGCCGCGTCGTAGTGCGCCATCAGT3' (ABI Carlsbad, California, United States). Initial denaturation was $30 \mathrm{~s}$ at $98^{\circ} \mathrm{C}$. Each cycle was $10 \mathrm{~s}$ at $98^{\circ} \mathrm{C}$, $30 \mathrm{~s}$ at $50^{\circ} \mathrm{C}$ and $30 \mathrm{~s}$ at $68^{\circ} \mathrm{C}$. PCR products were sizeselected on a $4 \%$ NuSieve 3:1 agarose gel followed by QIAquick gel extraction. To add a T7 promoter, sizeselected PCR products were re-amplified as above using the forward primer 5'-GGATTCTAATACGACTCAC TATACGCTCAGCGGCCGCAGCATCACCGCCAT CAGT-3'. Qiagen-purified PCR product was used as 
template for whole genome biotinylated RNA bait preparation with the MEGAshortscript T7 kit (Ambion) [13].

\section{Hybrid selection}

Hybrid selection using either synthetic bait or WGB was carried out as described previously [13]. Hybridization was conducted at $65^{\circ} \mathrm{C}$ for $66 \mathrm{~h}$ with $2 \mu \mathrm{g}$ of 'pond' libraries carrying standard or indexed Illumina paired-end adapter sequences and $500 \mathrm{ng}$ of bait in a volume of $30 \mu \mathrm{l}$. After hybridization, captured DNA was pulled down using streptavidin Dynabeads (Invitrogen Carlsbad, California, United States). Beads were washed once at room temperature for 15 minutes with $0.5 \mathrm{ml} 1 \times \mathrm{SSC} / 0.1 \% \mathrm{SDS}$, followed by three 10 -minute washes at $65^{\circ} \mathrm{C}$ with $0.5 \mathrm{ml}$ pre-warmed $0.1 \times \mathrm{SSC} /$ $0.1 \%$ SDS, re-suspending the beads once at each washing step. Hybrid-selected DNA was eluted with $50 \mu \mathrm{l}$ $0.1 \mathrm{M} \mathrm{NaOH}$. After 10 minutes at room temperature, the beads were pulled down, the supernatant transferred to a tube containing $70 \mu \mathrm{l}$ of $1 \mathrm{M}$ Tris- $\mathrm{HCl}, \mathrm{pH}$ 7.5 , and the neutralized DNA desalted and concentrated on a QIAquick MinElute column and eluted in $20 \mu \mathrm{l}$.

\section{Quantitative PCR enrichment measurement}

Enrichment of malaria DNA in samples was assessed using a panel of malaria qPCR primers designed to conserved regions of the P. falciparum 3D7 v.5.0 reference genome. Enrichment for each amplicon was calculated as the ratio between the amount of DNA presented preand post-hybrid selection, with threshold cycle (cT) counts corrected for qPCR efficiency using a standard curve for each amplicon. All qPCR reactions utilized 1 $\mu \mathrm{l}$ of template containing $1 \mathrm{ng}$ of total DNA. Estimated enrichment for the samples was calculated as the mean enrichment observed across all tested amplicons. Primer sequences and locations are listed in Additional file 3. Quantification of human DNA in the clinical samples was performed prior to sequencing using the Taqman RNase P Detection Reagents kit (Applied Biosystems Carlsbad, California, United States).

\section{Sequencing}

Each sample was sequenced at the Broad Institute using one lane of Illumina 76-bp paired-end reads. The libraries of pure P. falciparum DNA and hybrid-selected artificial clinical samples were each sequenced with one Illumina GAIIx lane. The hybrid-selected authentic clinical sample (Th231.08) was sequenced with one Illumina HiSeq lane. Sequence data have been deposited in the NCBI Short Read Archive under accession number [SRA029706].

\section{Analysis}

Quality scores on Illumina reads were rescaled using the MAQ sol2sanger utility [17]. Reads were then aligned to P. falciparum 3D7 (PlasmoDB 5.0) using BWA [18]. Sequenced reads were sorted and the consensus sequence was determined using the SAMtools utilities [19]. \%GC was calculated from 140-bp windows across the $P$. falciparum genome.

The human:P. falciparum DNA ratio in each sequence dataset was estimated from sequencing data by randomly sampling $50 \mathrm{~K}$ pairs of mated reads and measuring the fractions that uniquely mapped to human versus $P$. falciparum reference genome assemblies.

Simulated sequencing read coverage for the mock clinical sample prior to hybrid selection was performed by randomly sampling $1 \%$ of the read data generated for the pure $P$. falciparum sample, under the tested assumption that read coverage scales closely with parasite DNA fraction.

Principal components analysis was performed using Eigensoft software [20] on 8,300 non-singleton SNPs with coverage of at least 10 -fold in all strains and consensus quality scores of at least 30 .

\section{Additional material}

Additional file 1: Sequencing coverage comparison for $10-\mathrm{kb}$ genomic windows.

Additional file 2: Genomic locations of Agilent synthetic baits. Additional file 3: $P$. falciparum qPCR primers and locations (3D7 v.5.0 assembly).

\section{Abbreviations}

bp: base pair; qPCR: quantitative polymerase chain reaction; SNP: single nucleotide polymorphism; WGA: whole genome amplification; WGB: whole genome bait.

\section{Acknowledgements}

This project has been funded in part with Federal funds from the National Institute of Allergy and Infectious Diseases National Institutes of Health, Department of Health and Human Services, under contract number HHSN27220090018C. Funding was also supplied by a Global Health Program grant (number 49764) from the Bill and Melinda Gates Foundation and a grant from the National Human Genome Research Institute (number HG03067-05). We thank the Broad sequencing platform for sequence data generation.

\section{Author details}

'Genome Sequencing and Analysis Program, Broad Institute, 7 Cambridge Center, Cambridge, MA 02142, USA. ²Department of Immunology and Infectious Disease, Harvard School of Public Health, 665 Huntington Ave, Boston, MA 02115, USA. ${ }^{3}$ Faculty of Medicine and Pharmacy, Cheikh Anta Diop University, BP 7325, Dakar, Senegal.

\section{Authors' contributions}

AM designed and performed the experiments, wrote the manuscript, edited the manuscript and reviewed the data. PR designed and performed the experiments. AG designed and performed the experiments, supervised the project, edited the manuscript and reviewed the data. KG performed 
bioinformatic analyses, edited the manuscript and reviewed the data. TF performed bioinformatic analyses. DEN performed bioinformatic analyses, supervised the project, conceived and initiated the project and wrote the manuscript. DN and PDS provided samples. DVT, KGB and RD performed DNA extractions on the samples. JB coordinated sequencing. CR and DFW supervised the project. SKV, CN and BWB supervised the project, edited the manuscript and reviewed the data. All authors have read and approved the manuscript for publication.

\section{Competing interests}

The authors disclose that they are seeking to patent this application of hybrid selection and whole genome bait preparation.

Received: 3 May 2011 Revised: 22 July 2011 Accepted: 11 August 2011 Published: 11 August 2011

\section{References}

1. Lienau EK, Strain E, Wang C, Zheng J, Ottesen AR, Keys CE, Hammack TS, Musser SM, Brown EW, Allard MW, Cao G, Meng J, Stones R: Identification of a salmonellosis outbreak by means of molecular sequencing. $N$ Engl J Med 2011, 364:981-982

2. Neafsey DE, Barker BM, Sharpton TJ, Stajich JE, Park DJ, Whiston E, Hung CY, McMahan C, White J, Sykes S, Heiman D, Young S, Zeng Q, Abouelleil A, Aftuck L, Bessette D, Brown A, FitzGerald M, Lui A, Macdonald JP, Priest M, Orbach MJ, Galgiani JN, Kirkland TN, Cole GT, Birren BW, Henn MR, Taylor JW, Rounsley SD: Population genomic sequencing of Coccidioides fungi reveals recent hybridization and transposon control. Genome Res 2010, 20:938-946.

3. Truman RW, Singh P, Sharma R, Busso P, Rougemont J, Paniz-Mondolfi A, Kapopoulou A, Brisse S, Scollard DM, Gillis TP, Cole ST: Probable zoonotic leprosy in the southern United States. N Engl J Med 2011, 364:1626-1633.

4. Chin C-S, Sorenson J, Harris JB, Robins WP, Charles RC, Jean-Charles RR, Bullard J, Webster DR, Kasarskis A, Peluso P, Paxinos EE, Yamaichi Y, Calderwood SB, Mekalanos JJ, Schadt EE, Waldor MK: The origin of the Haitian cholera outbreak strain. N Engl J Med 2011, 364:33-42.

5. Mu J, Myers RA, Jiang H, Liu S, Ricklefs S, Waisberg M, Chotivanich K, Wilairatana P, Krudsood S, White NJ, Udomsangpetch R, Cui L, Ho M, Ou F, Li H, Song J, Li G, Wang X, Seila S, Sokunthea S, Socheat D, Sturdevant DE, Porcella SF, Fairhurst RM, Wellems TE, Awadalla P, Su X-zhuan: Plasmodium falciparum genome-wide scans for positive selection, recombination hot spots and resistance to antimalarial drugs. Nat Genet 2010, 42:268-271.

6. Van Tyne D, Park DJ, Schaffner SF, Neafsey DE, Angelino E, Cortese JF, Barnes KG, Rosen DM, Lukens AK, Daniels RF, Milner DA, Johnson CA, Shlyakhter I, Grossman SR, Becker JS, Yamins D, Karlsson EK, Ndiaye D, Sarr O, Mboup S, Happi C, Furlotte NA, Eskin E, Kang HM, Hartl DL, Birren BW, Wiegand RC, Lander ES, Wirth DF, Volkman SK, Sabeti PC: Identification and functional validation of the novel antimalarial resistance locus PF10_0355 in Plasmodium falciparum. PLoS Genet 2011, 7:e1001383

7. Trager W, Jensen JB: Human malaria parasites in continuous culture. Science 1976, 193:673-675.

8. Mons B, Boorsma EG, Ramesar J, Janse CJ: Removal of leucocytes from malaria-infected blood using commercially available filters. Ann Trop Med Parasitol 1988, 82:621-623.

9. Williamson J, Cover B: Removal of white blood cells from gametocyte-, schizont-, trophozoite- and ring stages of Plasmodium falciparum. Trans $R$ Soc Trop Med Hyg 1971, 65:416.

10. Richards WH, Williams SG: The removal of leucocytes from malaria infected blood. Ann Trop Med Parasitol 1973, 67:249-250.

11. O'Neill SL, Pettigrew MM, Sinkins SP, Braig HR, Andreadis TG, Tesh RB: In vitro cultivation of Wolbachia pipientis in an Aedes albopictus cell line. Insect Mol Biol 1997, 6:33-39.

12. Rasgon JL, Gamston CE, Ren X: Survival of Wolbachia pipientis in cell-free medium. Appl Environ Microbiol 2006, 72:6934-6937.

13. Gnirke A, Melnikov A, Maguire J, Rogov P, LeProust EM, Brockman W, Fennell T, Giannoukos G, Fisher S, Russ C, Gabriel S, Jaffe DB, Lander ES, Nusbaum C: Solution hybrid selection with ultra-long oligonucleotides for massively parallel targeted sequencing. Nat Biotechnol 2009, 27:182-189.

14. Gardner MJ, Hall N, Fung E, White O, Berriman M, Hyman RW, Carlton JM, Pain A, Nelson KE, Bowman S, Paulsen IT, James K, Eisen JA, Rutherford K,
Salzberg SL, Craig A, Kyes S, Chan M-S, Nene V, Shallom SJ, Suh B, Peterson J, Angiuoli S, Pertea M, Allen J, Selengut J, Haft D, Mather MW, Vaidya $A B$, Martin DMA, et al: Genome sequence of the human malaria parasite Plasmodium falciparum. Nature 2002, 419:498-511.

15. Kent BN, Salichos L, Gibbons JG, Rokas A, Newton IL, Clark ME, Bordenstein SR: Complete bacteriophage transfer in a bacterial endosymbiont (Wolbachia) determined by targeted genome capture. Genome Biol Evol 2011, 3:209-18.

16. Singer VL, Jones $\amalg$, Yue ST, Haugland RP: Characterization of PicoGreen reagent and development of a fluorescence-based solution assay for double-stranded DNA quantitation. Anal Biochem 1997, 249:228-238.

17. Li H, Ruan J, Durbin R: Mapping short DNA sequencing reads and calling variants using mapping quality scores. Genome Res 2008, 18:1851-1858.

18. Li H, Durbin R: Fast and accurate short read alignment with BurrowsWheeler transform. Bioinformatics 2009, 25:1754-1760.

19. Li H, Handsaker B, Wysoker A, Fennell T, Ruan J, Homer N, Marth G Abecasis G, Durbin R: The Sequence Alignment/Map format and SAMtools. Bioinformatics 2009, 25:2078-2079.

20. Patterson N, Price AL, Reich D: Population structure and eigenanalysis. PLoS Genet 2006, 2:e190.

doi:10.1186/gb-2011-12-8-r73

Cite this article as: Melnikov et al:: Hybrid selection for sequencing pathogen genomes from clinical samples. Genome Biology 2011 12:R73.

\section{Submit your next manuscript to BioMed Central and take full advantage of:}

- Convenient online submission

- Thorough peer review

- No space constraints or color figure charges

- Immediate publication on acceptance

- Inclusion in PubMed, CAS, Scopus and Google Scholar

- Research which is freely available for redistribution

Submit your manuscript at www.biomedcentral.com/submit
C Biomed Central 